\title{
Civilisations
}

Revue internationale d'anthropologie et de sciences

humaines

$63 \mid 2014$

L'Ancestralité revisitée

\section{Quand les Indiens dangereux deviennent des Indiens en danger}

Ancestralité et différence culturelle chez les Kakataibo d'Amazonie péruvienne

\section{Magda Helena Dziubinska}

\section{Q OpenEdition}

\section{Journals}

\section{Édition électronique}

URL : http://journals.openedition.org/civilisations/3722

DOI : $10.4000 /$ civilisations.3722

ISSN : 2032-0442

\section{Éditeur}

Institut de sociologie de l'Université Libre de Bruxelles

\section{Édition imprimée}

Date de publication : 30 septembre 2014

Pagination : 143-160

ISSN : 0009-8140

\section{Référence électronique}

Magda Helena Dziubinska, « Quand les Indiens dangereux deviennent des Indiens en danger »,

Civilisations [En ligne], 63 | 2014, mis en ligne le 30 septembre 2018, consulté le 19 avril 2019. URL:

http://journals.openedition.org/civilisations/3722 ; DOI : 10.4000/civilisations.3722 


\title{
Quand les Indiens dangereux deviennent des Indiens en danger Ancestralité et différence culturelle chez les Kakataibo d'Amazonie péruvienne
}

\author{
Magda Helena DZIUBINSKA
}

\begin{abstract}
Résumé : Depuis plus de dix ans les Kakataibo, soutenus par deux ONG, plaident devant le gouvernement péruvien, pour la création de deux réserves territoriales en faveur des Indiens vivant en isolement. Ces derniers ont été identifiés comme étant des Kamano, des Indiens qui occupent une place singulière dans la cosmologie kakataibo. L'ambivalence de leur statut ontologique est dû au fait qu'ils ne se laissent pas voir, ce qui rend d'autant plus difficile la validation du projet territorial par le gouvernement. Cet article passe en revue l'évolution de la manière dont les Kakataibo perçoivent les Kamano, en confrontant deux images diamétralement opposées, chacune liée à une génération différente. Nous verrons aussi que la mise en place du projet territorial a permis aux Kakataibo de construire un réseau relationnel avec des agents étrangers qui ont joué un rôle important dans l'élaboration, non seulement du nouveau discours sur les Kamano, mais aussi de la visibilité ethnique du groupe. Il s'agira ici de saisir, à travers une approche pragmatique, les enjeux identitaires et culturels sous-jacents au projet des réserves.
\end{abstract}

Mots-clés : groupes en isolement volontaire, réserves indigènes, Kakataibo, ethnicité.

Abstract: For over ten years the Kakataibo people, supported by two NGOs, have advocated before the Peruvian government for the creation of two territorial reserves for groups living in voluntary isolation. These groups were identified as Kamano, indigenous who occupy a particular place in the Kakataibo cosmology. Their ontological status is quite ambivalent because they do not allow themselves to be seen by others, which makes the validation of the project by the government more difficult. This article reviews the evolution of how the Kakataibo perceive the Kamano by comparing two diametrically opposed perspectives, each linked to a different generation. We will also see how the pursuing of the territorial project helped Kakataibo build a network of relationships with foreign actors who have played an important role in the development of the new discourse on Kamano and have contributed to the ethnic visibility of the group. The aim here is to capture, in adopting a pragmatic approach, the cultural and identity issues underlying the territorial project.

Keywords: people in voluntary isolation, indigenous reserve, Kakataibo, ethnicity. 
B ien que le sujet des Kamano", décrits comme « des indiens nus vivant au fond de la forêt », n'occupe pas une place importante dans les conversations quotidiennes des Kakataibo, un ensemble de récits, standardisés dans leur forme et leur contenu, leur est consacré. Ces récits qu'on transmet et qu'on partage sans restriction avec les autres, sont des histoires rapportées de rencontres avec les Kamano qui ont eu lieu dans le passé. Certains d'entre eux évoquent les combats menés par les aïeux des Kakataibo contre ces êtres dépeints alors comme sauvages et dangereux. Néanmoins, plus récemment, à côté de cette représentation ancienne des Kamano en a surgi une nouvelle, moins négative, et cela malgré l'absence de nouveaux récits de rencontre. Dans ce nouveau discours, énoncé en présence de l'ethnologue surtout par les jeunes, généralement mieux intégrés à la société nationale et à l'univers urbain, les Kamano n'apparaissent plus comme des Indiens " dangereux », mais plutôt comme des Indiens « en danger ». Ils sont décrits comme des ancêtres vivants, très vulnérables, qu'il faudrait protéger à l'intérieur de réserves ${ }^{2}$.

Le groupe Kakataibo, de langue pano, compte aujourd'hui environ 3500 individus répartis en trois sous-ensembles sur les fleuves Aguaytía, San Alejandro et Sungaruyacu en Amazonie péruvienne. Ces sous-ensembles régionaux correspondent aux trois sections dialectales différentes qui ont été décrites pour la première fois par Günter Tessmann en 1930. Dans cette même étude, Tessmann parle de deux Indiens Nokamán qu'il a rencontrés dans une hacienda sur le fleuve Ucayali, dans laquelle vivaient également d'autres Kakataibo, et dont le nom semble être une inversion du nom Kamano. Bien qu'aucun lien n'ait été fait par le chercheur allemand entre ces deux Indiens et les autres Kakataibo, le vocabulaire réuni par Tessmann atteste d'une affinité linguistique évidente entre eux et suggère qu'il s'agissait d'un dialecte de la même langue. Cela a été confirmé par Olive Shell (1975), linguiste du SIL, ainsi que par sa collègue Lila Wistrand (1969). Cette dernière ne parle toutefois pas de Nokamán mais de Kamano et constate qu'il s'agissait de : "many scattered, small groups living only on small inland streams rather than on the main rivers. Many of these latter groups are now extinct » (Ibid. : 147). Il convient de préciser que Tessmann est la seule personne à avoir rencontré les Nokamán, qui n'apparaissent à ma connaissance dans aucune autre source. Dans la mesure où le chercheur n'a visité aucun village de ce qu'il croit être un groupe Nokamán, et avance par ailleurs que celui-ci à l'époque de son voyage a été constitué de trois membres (Tessmann 1999 : 99), ces informations devraient être considérées avec beaucoup de prudence.

1 Kaman $=$ chien sauvage, $n o=$ ennemi. On les appelle également $«$ calatos $»=$ nus.

2 Toutes mes données ethnographiques ont été recueillies entre mars 2009 et septembre 2012, au cours d'un travail de terrain de dix-sept mois financé par l'aide au terrain du Legs Lelong et l'aide à la mobilité de l'Institut français d'études andines. Mes enquêtes ont été menées principalement dans les communautés kakataibo de Mariscal Cáceres et Yamino, ainsi que dans la communauté shipibo de Santa Rosa. 
Au début des années 1950, les missionnaires du Summer Institute of Linguistics $(\mathrm{SIL})^{3}$ se sont installés parmi les Kakataibo. Peu après la première école a été créée, et depuis un contact régulier s'est établi également avec la société nationale. L'incorporation des Métis à la communauté par le biais des intermariages, ainsi que la mobilité des jeunes Kakataibo qui partent de plus en plus souvent faire leurs études en ville ont accéléré l'intégration des Kakataibo à la société péruvienne et l'abandon des pratiques «traditionnelles ». Pour répondre à la forte demande d' " ethnicité visible » venue de l'extérieur, à laquelle doivent faire face aujourd'hui les groupes indigènes (Sahlins 1993 ; Galinier et Molinié 2006 ; Comaroff 2009), les Kakataibo sont en quête des traits culturels qui pourraient devenir emblématiques de leur autochtonie.

En 2005 la Federación Nativa de Comunidades Cacataibo (FENACOCA) ${ }^{4}$, soutenue par deux ONG laïques, l'Instituto del Bien Común (IBC) ${ }^{5}$ et le Centro de Conservación, Investigación y Manejo de Áreas Naturales (CIMA), a proposé au gouvernement péruvien un projet de création de deux réserves territoriales pour les Indiens vivant en isolement volontaire ${ }^{6}$. Selon la loi péruvienne relative à la création de réserves indigènes, les Indiens vivant en isolement volontaire sont ceux qui «n'ont pas développé de relations soutenues avec le reste de la société nationale, ou qui l'ont fait et ont décidé de les interrompre ». Comme on peut le voir sur la carte (Figure 1), le territoire de l'une des deux réserves proposées s'étend entre le côté oriental du Parque National Cordillera Azul et les deux communautés kakataibo Yamino et Mariscal Cáceres, tandis que la seconde serait adjacente à la communauté Puerto Azul, en haut du fleuve Aguaytía. Le Parque National Cordillera Azul s'étend sur un territoire de 1353 190,85 hectares entre les départements San Martín, Loreto, Ucayali et Huánuco et, depuis 2001, le CIMA est responsable de la gestion de l'ensemble des actions ayant pour but la préservation de sa richesse écologique. La partie sud-est du Parque était autrefois habitée par les aïeux des Kakataibo.

Dans les deux zones, l'exploitation du bois se fait à très large échelle et on y réalise depuis plusieurs années de la prospection pétrolière. De nombreux Kakataibo participent d'ailleurs à ces entreprises en tant que main-d'œuvre salariée. Bien qu'aujourd'hui cela soit impossible à confirmer, l'IBC ainsi que le CIMA soutiennent que les Indiens vivant en isolement volontaire au sud du Parque National Cordillera Azul, appartiennent au groupe Kakataibo. Ils les identifient aux Kamano, des Indiens qui, comme je vais le montrer, occupent une place très particulière dans l'imaginaire kakataibo.

3 Le Summer Institute of Linguistics a été fondé en 1934 aux États-Unis par des missionnaires protestants. L'Institut a entrepris l'évangélisation des populations indigènes à travers le travail linguistique (traduction de la Bible dans les différentes langues autochtones, alphabétisation des indigènes). En 1952 le gouvernement péruvien a chargé le SIL de l'éducation bilingue des différents groupes amazoniens. Les missionnaires nord-américains ont ainsi ouvert les premières écoles dans les communautés et assuré la formation des instituteurs indigènes.

4 La Federación Nativa de Comunidades Cacataibo a été créée à Aguaytía à la fin des années 1970 par Washington Bolivar, elle représente politiquement toutes les communautés kakataibo.

5 L'Instituto del Bien Común apporte un soutien juridique aux nombreux groupes indigènes du Pérou dans les différents projets liés à la reconnaissance légale de leurs terres, à la préservation de l'environnement et à la promotion du développement durable.

6 «Propuesta de Creación de dos Reservas Territoriales a Favor de los Cacataibos en Aislamiento» (2005) qui est une revitalisation d'une première demande formulée en 1999 par l'AIDESEP. 
Figure 1. Les communautés kakataibo et les plans des réserves pour les Indiens vivant en isolement volontaire.

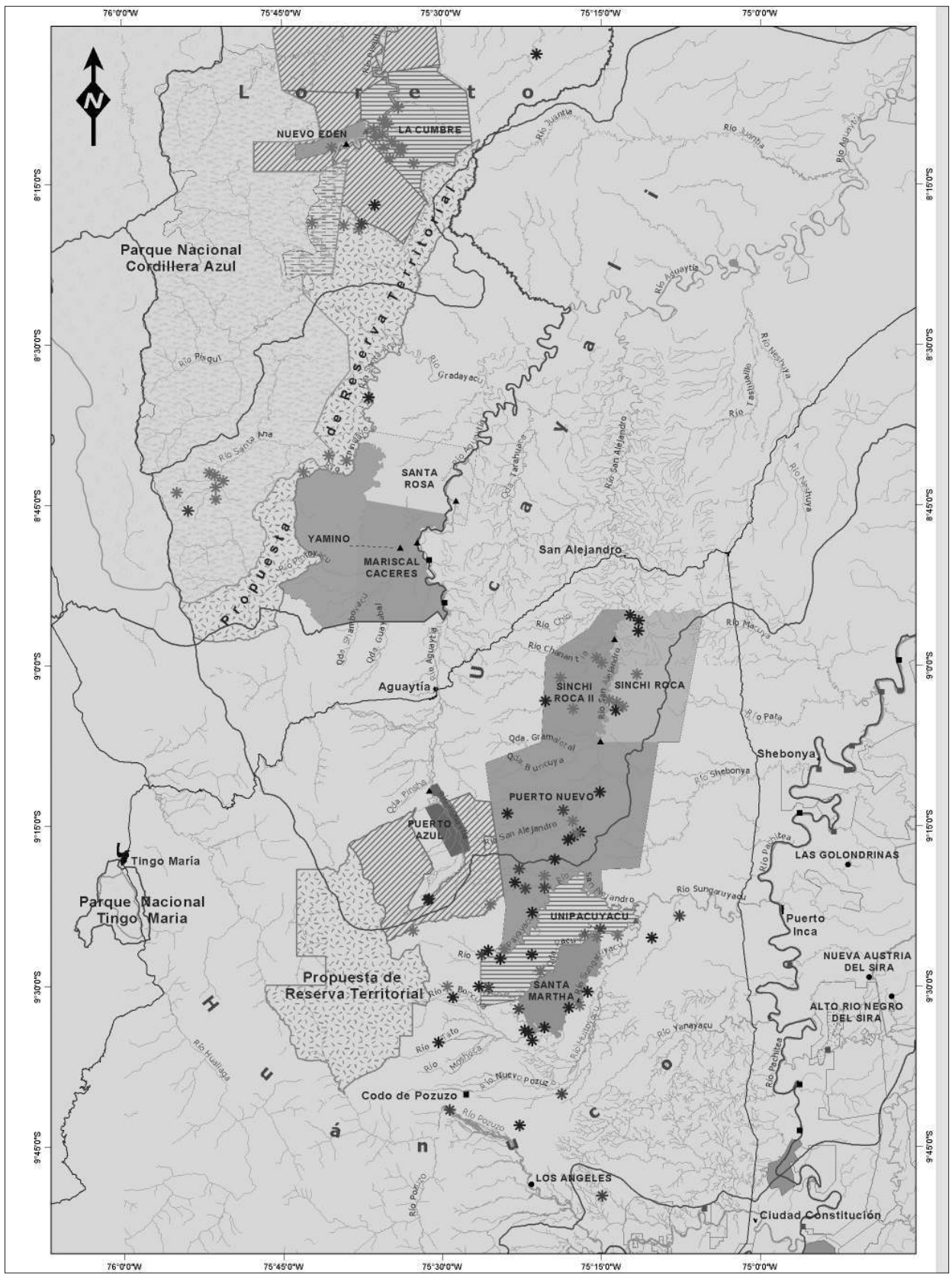

Source : Instituto del Bien Común (Lima). 
L'objectif de cet article est de réfléchir à la façon dont a émergé parmi les Kakataibo une nouvelle image des Kamano en tant que figures incarnant l'ancestralité et ayant besoin d'être protégées, en la situant dans le contexte sociopolitique plus global

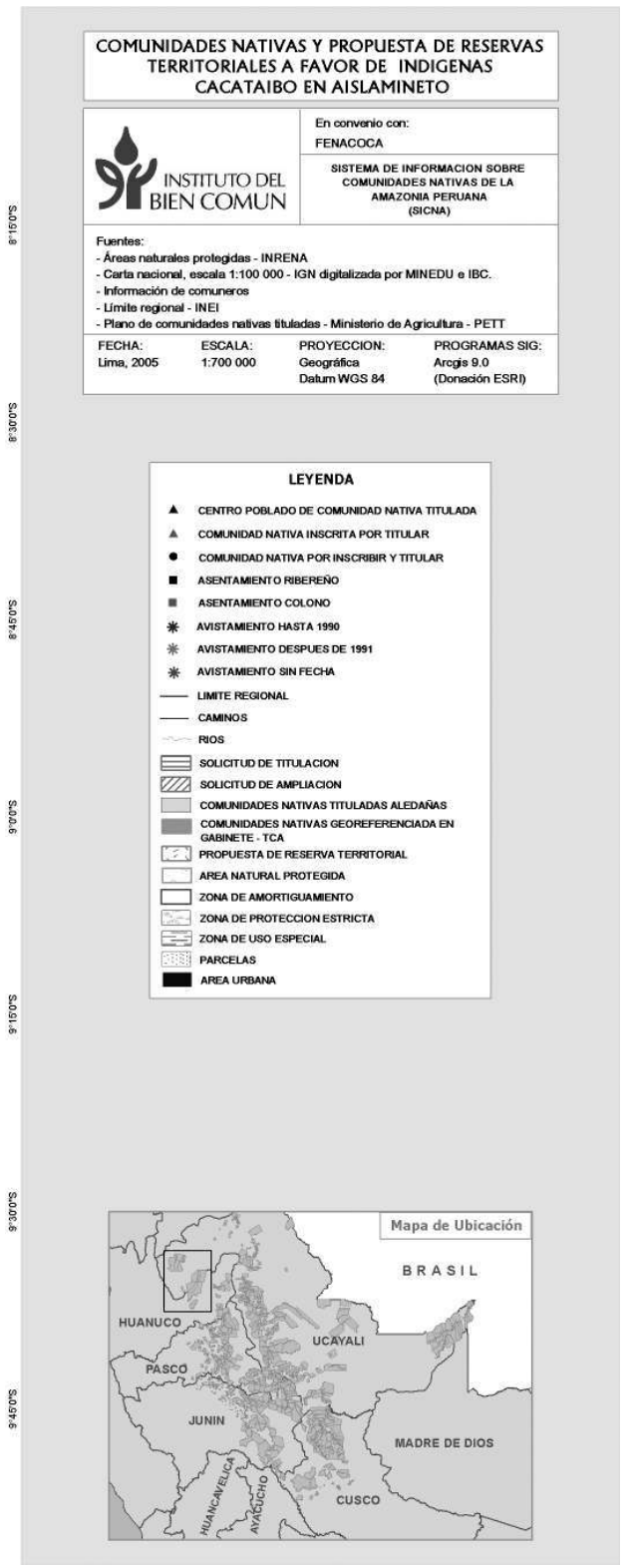
dans lequel vivent aujourd'hui les groupes amérindiens, en quête d'une reconnaissance aussi bien sur la scène nationale qu'internationale. La référence aux ancêtres n'est pas ici anodine et elle prête à une certaine confusion. En effet les Kakataibo qui, comme bien d'autres groupes amazoniens, pratiquent l'amnésie généalogique et s'efforcent d'effacer les traces des défunts, associent les morts et les ancêtres aux ennemis (Clastres 1968 ; Carneiro da Cunha 1975 ; Viveiros de Castro 1992). Cette représentation contraste donc avec le modèle historique de bien des sociétés africaines, orienté vers le culte des ancêtres (Fortes 1965 ; Kopytoff 1971). Toutefois l'idiome de l'ancestralité mobilisé aujourd'hui par les jeunes Kakataibo, renvoie plutôt à la logique patrimoniale où la référence aux origines a un potentiel de légitimation et peut être instrumentalisée pour appuyer certaines revendications identitaires, politiques et culturelles.

Mon hypothèse est que la demande de création de réserves indigènes pour les Kamano, plus que l'expression d'une réelle préoccupation des Kakataibo pour les Indiens en isolement, fait partie d'un projet plus grand de construction d'une nouvelle identité ethnique kakataibo. Dans un premier temps, je vais présenter les Kamano à partir des récits que j'ai recueillis pendant mon travail de terrain et des témoignages collectés par l'IBC en 2003. Le lien entre les Kamano, les ancêtres et les âmes des morts sera alors mis en évidence. Dans un deuxième temps, il sera question d'analyser le nouveau langage " protecteur » à l'adresse des Kamano, que les ONG ont introduit parmi les Kakataibo. Je terminerai par une réflexion sur les enjeux que la création de la réserve représente pour les Kakataibo, au-delà de 
l'enjeu territorial. Pourrait-on voir les Indiens isolés comme une sorte de patrimoine culturel ?

\section{Les Kamano : des « ancêtres vivants »}

Il y a un contraste frappant entre le fait que pendant mon travail de terrain je n'ai rencontré aucun Kakataibo qui aurait vu personnellement un Kamano et la cohérence avec laquelle ils décrivent ces êtres. Les Kamano incarnent d'abord une figure d'altérité et se distinguent des Kakataibo par une série de traits relevant tout autant de l'ordre physiologique, que linguistique ou moral. La différence est inscrite sur le corps même des Kamano : ce sont des êtres d'une taille imposante, ils ont de grands pieds, de longs cheveux parfois blonds, et la peau plus claire que celle des Kakataibo ${ }^{7}$. Mes hôtes, Emilia $^{8}$ (68 ans) et Gustavo (78 ans) qui appartiennent donc à la génération la plus âgée de la communauté Mariscal Cáceres, expliquent que le fait de vivre cachés au fond de la forêt $(n i)$ où le soleil les atteint très rarement, permet aux Kamano d'avoir « la peau des Blancs ». Eduardo, un octogénaire du même village, a insisté sur la beauté des femmes Kamano, qui sont selon lui « grandes, rondes et belles comme les femmes Blanches ». Puisqu'ils sont sauvages, les Kamano ne portent pas de vêtements, ils utilisent uniquement des morceaux de tissu en coton noués autour des hanches. Bien que la distance linguistique entre les Kamano et les Kakataibo soit régulièrement mise en avant par mes interlocuteurs, les opinions sur l'intercompréhension sont divergentes. Tandis que Gustavo, en racontant une rencontre inopinée entre un chasseur Kakataibo et les Kamano, constate : « on peut les comprendre, mais leur langue c'est juste des rhé-rhé-rhé », Emilia au contraire, insiste sur l'impossibilité de communiquer avec les Kamano. L'écart linguistique serait même la raison pour laquelle les Kakataibo n'ont pas pu les civiliser : « comment les sortir vers la lumière sans pouvoir communiquer avec eux ? $»^{9}-$ m'a demandé mon interlocutrice. Les plus âgés des Kakataibo évoquent de multiples tentatives pour civiliser, ou plutôt apprivoiser (raëa-), les Kamano qui se sont toutes soldées par un échec. Comme me l'a raconté Eduardo à Mariscal Cáceres : « Je ne les ai pas vus, mais mon père les a rencontrés. Mais ils l'ont fait courir avec leur pijayo $^{10}$ ! Il m'a dit qu'il leur laissait dans la forêt des vêtements pour qu'ils s'habillent, une casserole, un miroir pour qu'ils voient leur visage ${ }^{11} \ldots$ Ils sont très dangereux,

7 Les Piro décrivent les Yaminahua « sauvages » d'une manière fort similaire (Gow 1993: 330).

8 Les prénoms de mes interlocuteurs ont été changés pour protéger leur anonymat.

9 Bien que la « civilisation » renvoie à un complexe processus de transformation qui touche les différentes sphères de la vie des Kakataibo, elle est tout d'abord comprise dans sa dimension spatiale, comme la sortie du « fond de la forêt » et l'installation au bord d'un grand fleuve. Mes interlocuteurs ont beaucoup insisté sur cet aspect dans leurs narrations, en disant que leurs ancêtres voulaient aller vivre là « où il fait plus clair », ou qu'ils ne voulaient pas « vivre toute leur vie dans l'obscurité ».

10 Il s'agit du palmier bactris (Bactris Gasipaes) dont la particularité est qu'il ne pousse pas à l'état sauvage. Un arbre emblématique non seulement pour l'Amazonie, mais aussi pour la région andine. Dans le passé, les Kakataibo utilisaient les branches du bactris pour produire des flèches.

11 Une méthode classique utilisée par les missionnaires pour pacifier les Indiens non-contactés. Elle consistait précisément à déposer régulièrement toutes sortes de cadeaux (objets manufacturés) à proximité de leur emplacement jusqu'à ce que, séduits, ils commencent eux-mêmes à chercher le contact. 
ils tuent dès qu'ils voient quelqu'un s'approcher d'eux ». Nous voyons qu'en ce qui concerne la génération des Kakataibo les plus âgés, les Kamano sont associés la plupart du temps à la figure de l'ennemi (no) et un septuagénaire se souvient même qu'étant enfant il a appris à utiliser l'arc en visant le crâne d'un Kamano que son père a apporté d'une expédition comme trophée. Il semble que les attaques contre les Kamano se soient accélérées à l'époque du caoutchouc. Dans les années 1940, les Kakataibo du Bas Aguaytía, unifiés par Bolivar Odicio ${ }^{12}$, étaient payés par les patrons pour apporter de nouveaux esclaves. J'insiste sur le fait que, dans ce contexte, le terme "Kamano » désignait de nombreuses unités parentales habitant l'interfluve, qui refusaient de se joindre au groupe d'Odicio. Il ne s'agissait donc pas d'une catégorie figée qui aurait décrit un groupe particulier, mais plutôt d'un terme générique. Il arrive encore aux plus âgés parmi les Kakataibo du Bas Aguaytía de parler de l'histoire de leurs homologues des fleuves San Alejandro ou Sungaruyacu, en disant qu'autrefois ils étaient des kamano $^{13}$. Certains d'entre eux étaient donc incorporés à ce qu'est le groupe Kakataibo aujourd'hui, de nombreux étaient décimés par la chasse aux esclaves et les épidémies (Wistrand 1969 : 147). Il reste à savoir s'il y en a d'autres qui vivent jusqu'à présent en isolement.

Dans toute la littérature ethnographique, somme toute modeste, consacrée aux Kakataibo, les Kamano apparaissent systématiquement comme des êtres dangereux et malins, raison pour laquelle ils étaient combattus. Olive Shell, linguiste du SIL qui a travaillé avec les Kakataibo d'Aguaytía à la fin des années 1950, a enregistré un récit d'une attaque perpétrée contre les Kamano. Durant l'affrontement, les guerriers Kakataibo ont blessé un Kamano à la jambe. Sans pouvoir courir, il s'est caché dans un trou en laissant toutefois un pied dehors. Les Kakataibo ont essayé de le sortir en le tirant par le pied, sans succès. Finalement, ils ont décidé de le lui couper, en laissant ensuite le Kamano dans le trou avec une seule jambe (Shell 2008 : 13-14). Le lien avec les êtres de la forêt Chapchicë Tsabë évoqués par Erwin Frank (1994 : 199) semble évident. Ce qui caractérise ces personnages, c'est leur habitude fâcheuse de voler les conjoints Kakataibo afin d'avoir des relations sexuelles avec eux ; ce qui les différencie physiquement des êtres humains est précisément de n'avoir qu'un seul pied (Ibid.). Cela rejoint en partie ce que j'ai pu moi-même entendre sur les Kamano : pendant la nuit, ils volent les fruits des jardins des Kakataibo ; ils essayent aussi de capturer leurs femmes et, surtout, ils tuent tous ceux qui se trouvent sur leur chemin. Pour cette raison, il est fortement déconseillé de se déplacer seul dans la forêt. D'ailleurs, comme on le verra plus tard, les Kamano ne représentent pas le seul danger d'une promenade solitaire.

12 Né en Bas Aguaytía, Bolivar Odicio a été enlevé à l'âge de dix ans par les Shipibo-Conibo et probablement revendu en tant qu'esclave à César Odicio. Il a grandi dans son hacienda sur le fleuve Ucayali. Arrivé à l'âge adulte, le jeune Kakataibo a été vraisemblablement utilisé par le patron pour « unifier » par la force tous les Kakataibo dispersés sur les différents fleuves. Le seul objectif de cette entreprise fut évidement d'accroître l'effectif de la main-d'œuvre dont il avait besoin pour l'extraction du caoutchouc, de salsepareilles et du bois.

13 Le lien entre les Kamano et les Kakataibo de San Alejandro est également indiqué par le linguiste Roberto Zariquiey Biondi (2013) dans son étude comparative approfondie des dialectes kakataibo parlés aujourd'hui avec ceux décrits par Tessmann (1930). 
Les Kamano renvoient à l'ancestralité et à un temps révolu. "Les Kamano sont comme nos ancêtres », peut-on souvent entendre dire les Kakataibo à qui on demande de les décrire. Ils vivent en petits groupes et partagent la même maloca dont le toit est fait de palmes de yarina. Certains d'entre eux habitent dans de petites maisons provisoires (choza) que les Kakataibo ont encore l'habitude de construire dans leur jardin. Étant nomades, les Kamano se déplacent sans arrêt, ce qui rend leur rencontre d'autant plus difficile. Ils vivent de la chasse et de la pêche, mais il arrive parfois aussi qu'ils possèdent un petit jardin où ils cultivent des bananes plantain, de la patate douce, du maïs et du manioc. Comme les aïeux des Kakataibo, les Kamano produisent eux-mêmes les objets dont ils ont besoin : des haches en pierre, des flèches, de la vaisselle en terre cuite. Pour tromper l'ennemi, ils soufflent (xunka-) afin de l'endormir, comme le faisaient les guerriers Kakataibo autrefois. Ce qui donc rapproche les Kamano des ancêtres, et parallèlement les oppose radicalement aux Kakataibo d'aujourd'hui, c'est l'ignorance de la modernité. Qu'est-ce que la modernité aux yeux des Kakataibo ? Très brièvement, il s'agit de l'incorporation d'objets, d'institutions et de comportements appartenant au monde des Blancs : le sucre, le savon, le téléphone portable, les vêtements, l'école, la cumbia, les médicaments, le football : toute une panoplie moderne qui à présent fait partie de leur quotidien. En vivant isolés, les Kamano n'ont pas accès à cette richesse, une situation qui est perçue par les Kakataibo d'une manière ambivalente. D'un côté, elle a permis aux Kamano de conserver $"$ leurs coutumes ${ }^{14}$, mais de l'autre elle est quelquefois interprétée en termes de «souffrance » (tënëti). En général, les Kakataibo sont très friands d'objets manufacturés dont la possession représente une certaine intégration à la société nationale. Néanmoins, cette intégration ne devrait pas être comprise comme l'ambition d'être assimilés à des Métis. Il s'agit plutôt du désir de « contrôler » cette civilisation et le progrès, ou comme l'avait dit Sahlins (2007), d'indigéniser la modernité. Certes, mes amis Kakataibo reconnaissent volontairement que la possession d'objets de Blancs peut rendre la vie plus facile et plus confortable, mais c'est seulement en respectant les coutumes kakataibo, dont l'essentiel se résume aux conseils donnés par les plus âgés aux plus jeunes ('ësëti), que ces derniers sauront comment vivre bien et correctement.

Le mode de vie « à l'ancienne » est l'analogie la plus immédiate qu'on puisse établir entre les Kamano et les ancêtres, mais pas la seule. En effet, les deux catégories partagent certaines propriétés ontologiques.

\section{Les Indiens qui ne se laissent pas voir}

Certains de mes interlocuteurs Kakataibo soutiennent que les Kamano peuvent les observer, cachés par exemple au sommet des arbres, sans qu'eux-mêmes ne puissent les apercevoir. Ce constat revient à plusieurs reprises dans les témoignages recueillis par l'Instituto del Bien Común en 2003, lors de l'atelier consacré aux Indiens en isolement organisé dans toutes les communautés kakataibo ${ }^{15}$ : «ils sont en train de nous regarder,

14 En espagnol « sus costumbres », « sus creencias » (leurs coutumes ; leurs croyances).

15 Tous les témoignages sont retranscrits dans le document élaboré par l'IBC : "Propuesta de creación de dos reservas territoriales a favor de los Cacataibos en aislamiento », Instituto del Bien Común, Aguaytia-Lima, 2005. 
mais nous ne savons pas d'où ils nous regardent" (2005: 153), " même s'ils nous regardent, nous ne nous en rendons pas compte " (2005: 161), "nous n'avons pas essayé de communiquer avec eux, parce qu'ils ne se laissent pas voir » (2005: 165). Cette difficulté d'entrer en contact avec les Kamano est d'autant plus problématique que les témoignages oculaires directs sont indispensables pour légitimer la demande de création d'une réserve pour les Indiens isolés.

Ce qui empêche de voir les Kamano n'est pas seulement le fait qu'ils s'échappent au fond de la forêt dès qu'ils entendent le moindre bruit, mais aussi qu'il s'agit toujours d'une expérience visuelle modifiée. Le peu de témoignages directs recueillis par l'IBC, ainsi que celui décrit par María Cortez Mondragón (1987) ${ }^{16}$, suivent le schéma narratif que Pierre Déléage (2005) a identifié en analysant les récits de rencontres avec les esprits yoshi chez les Sharanahua, un autre groupe Pano. Les éléments essentiels de ces récits standardisés sont la solitude du témoin au moment de la rencontre et le brouillage de la perception provoqué la plupart du temps par la pluie ou la nuit ${ }^{17}$. Comme l'écrit Déléage :

[...] lorsque le paysage perceptif devient inhabituel, lorsque le savoir permettant de relier une perception, visuelle ou sonore, à une entité ontologique définie devient difficilement applicable. Dans de telles situations, le fait d'être seul, de ne pas pouvoir soumettre à autrui ses doutes, déclenche une forte angoisse : tous les ingrédients sont alors réunis qui permettront une rencontre avec un yoshi. (2005: 73)

Ou avec un Kamano ${ }^{18}$. Voici un récit recueilli par l'IBC qui reproduit fidèlement ce modèle narratif :

Ils étaient environ cinq cents personnes. Mon cousin pensait que ce sont nos frères de Puerto Nuevo en train de pêcher dans la source Guacamayo. Tout de suite il a eu très peur, ils faisaient un bruit énorme dans la forêt. Au moment où il a entendu ce bruit, une pluie torrentielle est tombée et on ne pouvait plus les voir. À ce moment, il a pensé, et quand quelqu'un se met à penser en entendant quelque chose de bizarre, sa tête se remplit de peur ${ }^{19}$, il pense qu'on va le tuer, et il ne peut plus les voir. (IBC $2005: 159)^{20}$

Dans ce cas-là, la perception du témoin a été doublement modifiée, d'abord par la pluie (qui a également transformé l'expérience auditive) et, deuxièmement, par

16 Toutefois, dans le même récit, le narrateur semble mélanger les témoignages de différentes personnes, en passant d'une narration à la première personne à une narration à la troisième.

17 Dans les récits kakataibo sur les rencontres avec les esprits ñushin (homologue de yoshi), la vision altérée est souvent provoquée par un état d'ivresse. Les personnes ivres sont considérées comme particulièrement faibles et vulnérables, ce qui en fait des proies d'autant plus faciles pour les esprits malins.

18 D'ailleurs, Déléage (2005 : 74) lui-même remarque qu'aujourd'hui les récits sur les yoshi, partagent le même schéma que ceux des Indiens non-contactés ou des narcotrafiquants qui se cachent dans la forêt.

19 «Penser vite» (sinan- bënë) est synonyme d'avoir peur en kakataibo.

20 Ma traduction depuis l'espagnol. 
la grande peur que la rencontre des Indiens sauvages provoque habituellement. Le motif de la peur revient dans les autres récits et, à chaque fois, elle empêche de voir clairement. L'informateur de Cortez Mondragón (1987 : 127) lui explique après avoir vu un Kamano : "Je me sentais comme ivre, mais j'ai vu, je me sentais m'évanouir en voyant une personne tellement bizarre, le corps devient nerveux ». Nous retrouvons avec ces récits le problème plus général du « voir » en Amazonie qui prend une importance toute particulière dans le contexte où il faut prouver l'existence des Indiens en isolement et délimiter la zone où ils sont supposés vivre. En effet, les histoires sur les Kamano que j'ai pu entendre de mes informateurs Kakataibo ne sont pas fondées sur une expérience ostensive personnelle, mais reproduisent les récits qui leur ont été racontés par leurs proches, sans jamais mettre en cause l'autorité de ces derniers, ni la véracité de leur histoire. En lisant le document de l'IBC, on ressent le malaise de la personne chargée de recueillir les témoignages face à cette imprécision des Kakataibo sur ce qu'ils ont $v u$. Le problème est que « voir » renvoie, dans de nombreux cas, au fait de percevoir certains signes ou certains phénomènes qui pourraient suggérer la présence des Kamano. Parmi ceux-ci, les plus fréquemment évoqués sont les empreintes laissées sur le sol, les « bruits bizarres » (les cris des Kamano, mais aussi le bruit des branches cassées) ou des objets qui disparaissent (et dont on suppose que les Kamano les ont volés). D'autre part, les Kakataibo reconnaissent deux manières de voir, nettement distinguées au plan linguistique. La première, exprimée par le verbe " is- ", qu'on pourrait traduire par « voir/regarder », et l'autre par le verbe «ñacha- », qui veut dire « voir bien/reconnaître quelqu'un $»^{21}$. Bien que les récits sur les Kamano aient été recueillis par l'IBC en espagnol, on ressent cette nuance sémantique.

Il y a une corrélation incontestable entre les Kamano et les esprits ñushin. Les uns comme les autres sont difficiles à voir et vivent au fond de la forêt. Ils ne se contentent pas d'effrayer leurs victimes, mais ils les blessent avec des flèches, en lançant des pierres ou en les passant à tabac. La forêt représente pour les Kakataibo tout ce qui est incontrôlable et dangereux. Elle est habitée par différents êtres malveillants, parmi lesquels il y a non seulement les Kamano et les ñushin, mais aussi les animaux sauvages, les Utano ${ }^{22}$ ou les Pishtaku ${ }^{23}$. Ils partagent beaucoup de traits communs et sont souvent confondus par les Kakataibo eux-mêmes. Frank (1994) met dans la même catégorie les voleurs d'époux Chapchicë Tsabë, les Kamano et les Utano, tous êtres qui, malgré leur apparence plus ou moins anthropomorphe, ne sont pas qualifiés de "vrais hommes » (uni). Ailleurs, Frank désigne les Kamano comme des « êtres mythiques » (1990 : 82). La confusion entre les Kamano et les Utano apparaît également dans l'article déjà

21 On utilise aussi ce mot pour prévenir quelqu'un, par exemple, lors de la marche dans la forêt, de bien regarder où il met les pieds, pour éviter les serpents.

22 Les Utano, appelés parfois ñushin ou « diables de la forêt », vivent également dans la forêt. Par contraste avec les Kamano, de taille imposante, ils se caractérisent par leur physionomie d'enfant. Malgré cette apparence enfantine, les Utano sont très dangereux. Ils capturent les femmes et les enfants non accompagnés qu'ils tuent si les membres de leur famille ne viennent pas les récupérer.

23 À l'origine, un personnage de la mythologie andine qui a imprégné certaines mythologies amazoniennes. Pishtaku qui a l'apparence d'un Blanc (les cheveux blonds et les yeux bleus), assassine les Indiens afin de prélever leur graisse. Selon les Kakataibo, aujourd'hui les Pishtaku enlèvent aussi les organes de leurs victimes indiennes pour pouvoir guérir les Blancs. 
cité ici de Cortez Mondragón (1987). Chacune de ces entités est un prédateur dont la rencontre provoque une peur immense qui, d'après les Kakataibo, peut causer la perte temporaire de l'âme et même la mort.

Revenons aux ancêtres. Au moment de la mort, l'âme du défunt, son bëru ñushin (l'esprit de l'œil) ${ }^{24}$ part vers le ciel (nai). Avant ce passage toutefois, elle rôde autour du village, d'où l'importance de garder la paix pendant la veillée mortuaire pour ne pas la fâcher. D'après mes hôtes, les âmes des morts ont peur de la solitude et demandent d'être accompagnées par les cognats vivants. Pour cela il est strictement interdit de quitter le village dans les jours qui suivent l'enterrement. Cela pourrait provoquer chez le mort un sentiment d'abandon et il pourrait vouloir se venger en emmenant quelqu'un avec lui. Après être monté dans le ciel, le bëru ñushin du défunt se mélange avec les autres âmes et devient rara ñushin, l'âme ancêtre. Bien qu'ils vivent dans le ciel, les ancêtres observent toujours les Kakataibo sur la terre. Plus encore, ils s'aventurent dans le monde des humains, pour leur faire peur ou pour essayer d'enlever leur âme. Ils attaquent leurs victimes dans leur sommeil ou lors de promenades solitaires dans la forêt. Cette faculté de surveillance post-mortem exercée par les ancêtres fait penser à ce que les Kakataibo disent des Kamano, à savoir qu'ils nous regardent tout le temps, sans qu'on sache vraiment d'où ils le font ${ }^{25}$. D'autre part, plusieurs Kakataibo ont indiqué que les Kamano se cachent, ou même vivent, sur les arbres, donc au-dessus de la terre, ce qui les rapproche encore une fois, spatialement cette fois-ci, des ancêtres.

Les Kakataibo ne sont pas les seuls à établir une corrélation entre les Indiens sauvages et les ancêtres. Le même lien a été fait par un autre groupe Pano, les Kashinawa (Keifenheim 1997). Toutefois, la stratégie qu'ils ont adoptée face à ces Indiens, appelés Mashiku, était tout à fait différente de celle des Kakataibo, de même que le rôle des acteurs externes dans la mise en place de ces stratégies. Dans le premier cas, le Summer Institute of Linguistics, souvent chargé d'établir le contact avec les Indiens isolés, a délégué cette tâche aux Kashinawa eux-mêmes, en leur fournissant des objets manufacturés pour attirer les Mashiku vers l'extérieur. En conséquence, les Kashinawa ont essayé de les capturer et les intégrer au groupe en tant qu'alliés ou esclaves (Ibid. : 152). La situation des Kakataibo est tout à fait différente. D'abord parce que ce sont des organisations étrangères qui dirigent le projet de création de réserves pour les Indiens en isolement et que c'est en grande partie à leur initiative que les Kakataibo sont devenus les avocats des Kamano ; deuxièmement parce qu'elles proscrivent toute tentative de prise de contact avec ces Indiens.

Ce que le matériel ethnographique lié aux Kamano laisse entrevoir en premier lieu, c'est l'ambiguïté ontologique de ces êtres. Cette ambiguïté est entretenue par les Kakataibo dans leurs discours, où se croisent différents registres : historique, mythique, imaginaire, politique. Il n'y a pas chez les Kakataibo un seul discours sur les Kamano : ils sont multiples et leur contenu dépend non seulement de l'identité et de l'âge de

24 Traduit par les Kakataibo en espagnol comme « alma » (âme).

25 Dans son article sur les esprits mariwin qui incarnent l'ancestralité chez les Matis, et qui sont personnifiés par les hommes masqués lors des rituels, Erikson (2004) insiste sur la brillance de l'œil du masque qui fait penser au bëru ñushin. Une contrainte liée au regard est imposée lors des mascarades rituelles : tandis que les enfants doivent éviter de regarder les esprits, ces derniers doivent constamment les fixer du regard. 
l'énonciateur, mais aussi du contexte de l'énonciation et de l'identité du destinataire. Jusqu'à maintenant j'ai essayé de saisir la façon dont les Kamano sont perçus par la génération la plus âgée des Kakataibo, vivant dans la communauté. À présent je voudrais aborder un autre discours sur ces Indiens, promu par les ONG, que l'ethnologue peut entendre surtout de la part des Kakataibo plus jeunes et davantage liés au milieu urbain.

\section{Le nouveau discours sur les Kamano}

Les Kamano sont présentés par les ONG comme des Indiens en danger, dont la survie est menacée par l'exploitation intensive des ressources naturelles (gaz, pétrole, bois) qui a lieu dans la région. Je souligne que de nombreux Kakataibo sont employés dans ces entreprises d'exploitation, y compris ceux qui se considèrent comme défenseurs de la cause des Kamano. Bien que les effets écologiques de ces activités soient encore mal compris par beaucoup de Kakataibo - un jeune homme engagé dans la vie politique m'a expliqué, par exemple, que les compagnies pétrolières disposent aujourd'hui « d'appareils modernes » qui n'ont aucun impact sur l'environnement - les effets sociaux sont immédiats. Comme dans d'autres parties de l'Amazonie, la présence d'entreprises forestières provoque de nombreux conflits internes car les accords sont négociés uniquement avec quelques personnes parmi les plus influentes du groupe, qui vivent habituellement en ville. Le reste des Kakataibo, exclus de l'affaire, seront seulement partiellement informés des conditions du contrat. Les sommes d'argent perçues par le chef $(a p u)$ sont en outre régulièrement escamotées et font d'autant plus facilement l'objet de spéculations et d'accusations dans la communauté (Dziubinska 2014).

Bien que le Pérou possède une législation spécifique qui, en théorie, devrait garantir la protection des Indiens non-contactés ou en isolement volontaire, ainsi que leur droit de décider du mode de vie qu'ils souhaitent mener, et protéger leur droit à la terre ${ }^{26}$, les contradictions qui leur sont propres rendent ces lois plutôt inopérantes. En effet, tout en reconnaissant son devoir de protéger les Indiens isolés en créant des « réserves indigènes », l'État autorise les activités «d'intérêt national » à l'intérieur de ces territoires. Parmi ces activités il y a l'exploitation pétrolière et forestière, dont on sait les effets néfastes sur l'environnement, qui pourraient avoir des conséquences désastreuses pour les Indiens en isolement. Il ne s'agit donc pas de zones strictement protégées et fermées aux entreprises d'exploitation (comme on pourrait s'y attendre), mais de réserves où la présence d'agents externes est soumise à des contrôles plus rigoureux.

26 Loi n 28736 promulguée le 24 avril 2006. L'État péruvien a également ratifié la Convention 169 de l'Organisation Internationale du Travail (une institution des Nations Unies) qui traite spécifiquement des droits des peuples indigènes, parmi lesquels ceux à l'autodétermination et à la terre. 
Figure 2. Une affiche du projet de création de réserves indigènes.

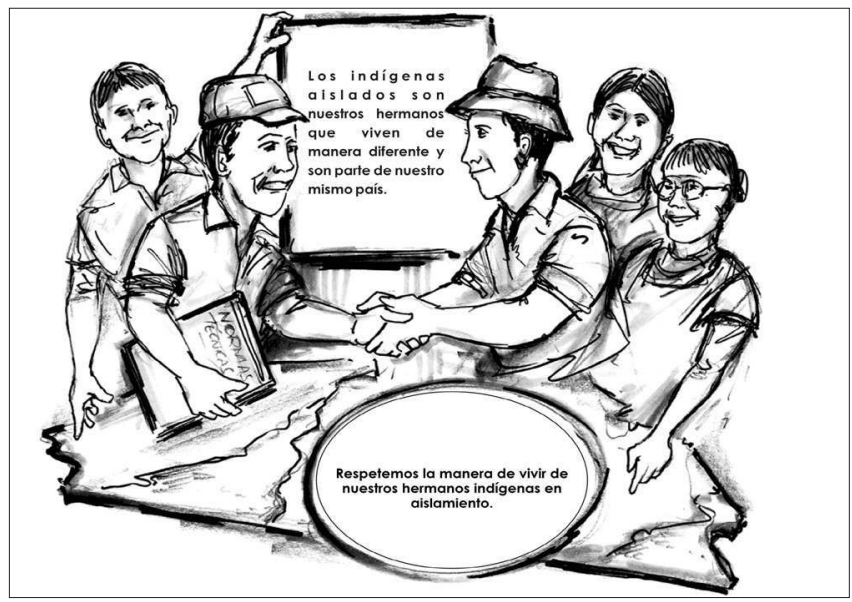

Source : www.cima.org.pe $e^{27}$

La demande de création de réserves pour les Indiens Kakataibo en isolement n'a jamais été acceptée par le gouvernement. Depuis plus de dix ans deux ONG (l'IBC et le CIMA) apportent une aide logistique à la Fédération kakataibo (FENACOCA), afin que sa requête territoriale soit entendue. Pour que les réserves indigènes puissent être créées, il faut d'abord prouver l'existence des Indiens en isolement. D'où la principale difficulté de cette entreprise car, d'un côté il y a très peu de témoignages directs sur les Kamano, et de l'autre le contact recherché avec ces Indiens est prohibé. La présence des ONG et l'élaboration du projet de réserves territoriales ont contribué toutefois à l'introduction parmi les Kakataibo d'un discours tout à fait nouveau concernant les Kamano. L'affiche crée par le CIMA (Figure 2) en est une bonne illustration.

Tout d'abord on ne parle plus d' " ennemis sauvages », mais de " frères », d'Indiens égaux aux Kakataibo, qui ont décidé de vivre en isolement. Un choix de vie auquel ils ont dorénavant le droit et qui devrait donc être respecté. Ce langage égalitaire et paternaliste contraste fortement avec la représentation ancienne que les Kakataibo avaient des Kamano, en les situant tout en bas de l'échelle des êtres, eux-mêmes occupant son sommet en tant qu'uni (les vrais hommes). La dangerosité des Kamano n'est pas complètement évacuée de leur nouvelle image. Le CIMA prévient dans ses prospectus et à travers les ateliers éducatifs que, voulant éviter le contact avec les étrangers, les Kamano peuvent attaquer les gens avec leurs flèches lors d'une rencontre inopinée. Toutefois, dans la nouvelle conjoncture, le risque est paradoxalement plus grand pour l'attaquant à cause de sa vulnérabilité. Le CIMA a même élaboré un « guide pratique » pour les Kakataibo, en expliquant comment se comporter en cas de rencontre imprévue avec un Kamano. Les conseils les plus importants sont les suivants : ne pas s'approcher; ne pas crier ; laisser les Kamano prendre tous les objets qu'ils voudraient

27 Cadre en haut : "Les indigènes en isolement sont nos frères qui vivent d'une manière différente et font partie de mon pays ». Cadre en bas : "Respectons la manière de vivre de nos frères indigènes en isolement $»$. 
(mais essayer tout de même de les désinfecter avant !) ; ne pas utiliser d'appareil photo ; s'ils veulent manger, leur donner un repas récemment cuisiné (éviter cependant les plats salés et épicés, inconnus des Kamano) ; isoler les individus visiblement malades ; observer le type de vêtements qu'ils portent ainsi que les marques corporelles (telles peintures et tatouages) (CIMA 2011 : 19). On voit bien que la nouvelle image des Kamano est une inversion symétrique de la représentation « traditionnelle » :

\begin{tabular}{|l|c|c|}
\hline & Ancien registre & Nouveau registre \\
\hline Position relationnelle & Ennemi (no) & Frère (xucën) \\
\hline Nature & Dangereux & Vulnérable \\
\hline Stratégie & Guerre (rapt, trophées) & Protection \\
\hline
\end{tabular}

Des personnes dont le statut ontologique n'est pas tout à fait certain (si on prend en compte ce que les Kakataibo en disent) se matérialisent à travers les nouvelles images et les nouvelles connaissances véhiculées par les ONG. Un jeune Kakataibo de 27 ans, employé à la FENACOCA a constaté : « il faut protéger les Kamano, pour qu'ils puissent vivre tranquillement, il faut les défendre, défendre leurs droits, pour que personne ne les dérange. Qui d'autre pensera à eux sinon?». Son ami ajoute : "nos frères Kamano ont le droit de vivre comme ils veulent, comme ils aiment, ils ont aussi le droit d'avoir leur terre, pour pouvoir y vivre, c'est pour cette raison qu'il faut les aider, c'est pour ça que nous faisons ce projet, pour que le gouvernement nous entende ». On aurait pourtant tort de penser que cet idiome est utilisé par tous les Kakataibo. En effet, la question des Kamano préoccupe surtout les jeunes hommes, éduqués, installés d'une manière plus ou moins permanente dans la ville et engagés dans la vie politique du groupe et dans la promotion de leur culture. Il s'agit d'un langage politique, que les jeunes Kakataibo utilisent pour parler d'eux avec des personnes extérieures au groupe, et surtout avec des Blancs. Ce type d'énoncé est complètement étranger aux plus âgés des Kakataibo.

La nouvelle perception des Kamano est tout à fait cohérente avec le caractère inclusif et appropriatif du système socio-cosmologique kakataibo. L'Autre, considéré la plupart du temps comme l'ennemi (no), représente dans ce contexte les ressources nécessaires à la production des personnes et à la reproduction du groupe en général ${ }^{28}$. Il était autrefois incorporé à travers la guerre qui embrassait chez les Kakataibo le modèle classique pour l'Amazonie, basé sur le rapt et la prise de trophées. La guerre n'est plus pratiquée depuis plusieurs dizaines d'années, mais l'altérité est toujours une composante essentielle des nouvelles constructions identitaires. Force est en effet de constater que les Kamano constituent une sorte de réservoir d'images fantasques de l'indianité à l' " état pur », que les Kakataibo se sont appropriées. Puisque l'élément natif représente un capital symbolique très recherché sur le marché ethnique actuel, ces images ont acquis un pouvoir exceptionnel en attirant l'attention des médias et des ONG. Je souhaite à présent mettre en lumière leur caractère performatif, car la question

28 Voir Erikson 1986, Viveiros de Castro 1992, Descola 1993, Vilaça 1999. 
des Kamano a permis aux Kakataibo d'établir un réseau relationnel avec des acteurs étrangers puissants, qui ont contribué notablement à forger la " culture » kakataibo objectivée et ethnicisée.

\section{La fabrique de l'indianité}

Quand, en 2007, j'ai annoncé à un chamane Shipibo-Conibo de la communauté San Francisco ma volonté de visiter les Kakataibo, celui-ci n'a pas caché son étonnement : " mais pourquoi veux-tu aller voir les Cashibo ${ }^{29}$ ? Il n'y a rien d'intéressant à voir làbas. Ils sont comme les Métis. Ils ont perdu toute leur culture. C'est pas intéressant làbas ». En effet, les Shipibo-Conibo symbolisent ce qu'on pourrait appeler une indianité idéale au Pérou. Leur art est reconnu et exposé dans les plus grands musées du monde entier $^{30}$, tandis que leurs chamanes, auxquels on attribue des pouvoirs particuliers, attirent un grand nombre d'Occidentaux en quête mystique voulant expérimenter le rituel d'ayahuasca. Il n'est donc pas étonnant que les Shipibo-Conibo aient établi une sorte de quasi-monopole sur l'indianité dans la région. Le désir d'une jeune ethnologue (supposée étudier la culture) d'aller séjourner parmi les Kakataibo (qui « ont perdu leur culture ») leur paraissait simplement absurde. Ce discours négatif sur les Kakataibo est intériorisé par ces derniers. En arrivant à Mariscal Cáceres, Emilia m’a confié : "Personne ne vient nous voir. Tout le monde pense que nous sommes des Métis. Mais ce sont les Shipibo qui ont raconté ces mensonges. Nous ne sommes pas Métis. Nous avons notre culture, nous parlons notre langue, nous avons nos chants, nos flèches ». La culture évoquée ici par le chamane Shipibo-Conibo et par Emilia est celle que Carneiro da Cunha (2010) met entre guillemets, en l'opposant à la culture (sans guillemets). Cette « culture » fait partie d'un système interethnique : « elle appartient à un métalangage, elle est une notion réflexive qui d'une certaine façon parle à propos d'elle-même» (Ibid. : 77). Pour de nombreuses sociétés locales, la "culture » ne représente pas un héritage, mais un " projet » de production interculturelle à l'échelle nationale et internationale. Bien qu'il soit souvent converti en marchandise, il fait ressortir en même temps son potentiel de résistance, d'émancipation et de revendication.

La protection de la biodiversité, des Indiens en isolement et de la « culture indigène » faisant partie d'une même rhétorique, le CIMA a parrainé différentes actions ayant pour but de promouvoir la culture kakataibo. L'une d'entre elles a été la création, en 2009, de l'Association des femmes artisanes Kakataibo des communautés de Yamino et de Mariscal Cáceres, qui ont été plusieurs fois invitées à participer à des foires indigènes à Lima, où elles ont pu montrer et vendre leur artisanat. J'ai été présente à Yamino lorsque les Kakataibo ont reçu la première invitation à ce genre d'événement. Elle a été accueillie avec un grand enthousiasme, le seul problème qui s'est posé, et qui s'est révélé des plus banals, étant... le manque d'artisanat ! Suite à une formidable mobilisation de toute la

29 Cashibo est le nom par lequel les Kakataibo sont appelés par leurs voisins et anciens ennemis ShipiboConibo. Dans les langues pano « cashi » signifie chauve-souris, une allusion claire au cannibalisme. Ce nom est considéré par les Kakataibo comme une insulte grave et il est aujourd'hui catégoriquement rejeté.

30 En 2008, 1'Instituto Nacional de Cultura Peruana a déclaré les dessins quéne shipibo-conibo patrimoine culturel de la nation. 
communauté, l'artisanat « traditionnel » a été autant réactivé qu'inventé et bricolé de toutes pièces en à peine deux semaines. Les femmes, les hommes, les enfants, les Métis, tout le monde présent au village s'est mis à travailler du matin au soir, à fabriquer des ceintures de graines, des couronnes, des flèches, mais aussi à peindre des toiles comme le font les Shipibo et à produire des colliers et des bracelets de style métis. Pour que tout soit « parfait », les artisans s'aidaient avec des règles et des crayons à papier ; les défauts ont été gommés; les peintures naturelles ont été combinées avec les couleurs des feutres ; une couturière métisse a été invitée pour enseigner aux femmes Kakataibo comment coudre des sacs à main avec poches et fermeture éclair qu'elles décoraient ensuite avec les dessins « traditionnels ». Une délégation de Kakataibo a été ensuite désignée pour se rendre à la capitale, accompagnée par le coordinateur du CIMA, afin d'exposer l'artisanat. Ils sont rentrés plutôt satisfaits en annonçant avec joie : « tout a été vendu ! » Le gain matériel mis à part, le fait de « tout vendre » signifie avant tout que ces nouvelles créations artistiques ont été en quelque sorte validées, gagnant ainsi leur légitimité. Le « Catalogue de l'Art Kakataibo » (2011) fut dès lors publié par le World Agroforestry Center, toujours en collaboration avec le CIMA. Il est le résultat d'un atelier dont l'objectif a été, comme l'on peut lire dans l'introduction au catalogue, d' « identifier des plantes localement importantes, [...] revitaliser l'identité culturelle kakataibo et contribuer à la diffusion des connaissances écologiques du groupe ». Cette (re)vitalisation de l'artisanat a entraîné un autre projet culturel très récent, inauguré en 2011, celui de la mise en tourisme de la communauté Yamino. Le projet touristique, soutenu cette fois-ci par la United States Agency for International Development, consistait à construire quatre malocas - de grandes maisons traditionnelles dans lesquelles habitaient les Kakataibo d'antan. Aujourd'hui en effet, les Kakataibo vivent dans des maisons plus petites de style métis et les malocas servent uniquement à accueillir des visiteurs étrangers. Elles pourront abriter aussi les événements culturels que les Kakataibo envisagent d'organiser dans le futur, et qui engendreront sans doute de nouvelles réadaptations culturelles. D'autre part, les habitants de Yamino expliquent que les femmes pourront y vendre leur artisanat et les plats typiques aux touristes qu'ils espèrent attirer. Lors de mon premier séjour parmi les Kakataibo en 2007, un jeune homme kakataibo, à l'époque membre de la FENACOCA, m'a également parlé d'un projet de création d'un centre chamanique pour les Blancs. L'idée m'a paru d'autant plus curieuse qu'il n'y avait pas de chamane dans la communauté. Mon interlocuteur a envisagé deux solutions à cet obstacle : soit « emprunter» les chamanes Shipibo-Conibo et les installer dans la communauté kakataibo, soit réaliser des « cours intensifs » auprès de ces chamanes et ensuite revenir pour faire bénéficier en toute autonomie les touristes de ces nouveaux savoirs.

Après avoir longtemps vécu à l'ombre des Shipibo-Conibo, la question des Kamano ramène les Kakataibo sur l'avant de la scène ethnique. Les exemples de productions culturelles que je viens d'évoquer font partie d'une stratégie de définition de soi, qui répond aux critères du monde qu'on appelle conventionnellement globalisé et à la vision de l'autochtonie qu'il impose. Celle-ci, nourrie autant par l'industrie touristique que par les politiques patrimonialisantes est par principe visible, exotique et de plus en plus souvent écologique et spiritualisée. Les nouvelles productions culturelles laissent surtout entrevoir le caractère fondamentalement interethnique de cette « culture ». Elle est autant le fruit de la créativité audacieuse des Kakataibo que des agents extérieurs 
à la communauté, qui donnent un élan à toute l'entreprise, dont les enjeux sont autant identitaires et culturels que politiques et économiques. C'est surtout dans ce contexte interactionnel que ces expressions artistiques prennent tout leur sens.

\section{Conclusion}

Dans cet article je me suis attachée à présenter les différentes représentations des Indiens sauvages Kamano qui existent parmi les Kakataibo. L'accent a été mis sur leur caractère polysémique et le statut ontologique ambivalent de ces êtres qui transparaît dans les récits. Tandis que les Kakataibo qui habitent dans la communauté décrivent les Kamano comme des ennemis sauvages et dangereux, les jeunes vivant en ville parlent de frères vulnérables dont les droits à la vie en isolement et à la terre doivent être défendus. Ces deux visions à première vue contradictoires, possèdent un élément commun : dans les deux cas les Kamano apparaissent comme des êtres demeurant en dehors du temps et de l'histoire, une sorte de fossiles vivants témoignant d'une époque depuis longtemps révolue. Bien que le second discours soit le fruit des interactions avec les ONG étrangères, les Kakataibo ont su se l'approprier pour servir leurs propres intérêts qui, on l'a vu, vont au-delà des seuls enjeux territoriaux. Dans le guide touristique de la région, élaboré en 2011 par la municipalité d'Aguaytía, sont citées parmi les lieux de grand intérêt les communautés kakataibo qui «ont conservé toutes leurs coutumes ancestrales ». Aussi imprécise qu'une telle affirmation puisse paraître, elle représente en tout cas un réel défi pour les Kakataibo d'aujourd'hui. Un défi qu'ils relèvent avec une extraordinaire rapidité et créativité. Les Kamano, auxquels on fait désormais référence dans tous les documents et dans tous les projets qui sont menés au sein du groupe Kakataibo, la construction de malocas, la réactivation des différentes techniques artisanales et les peintures corporelles que les Kakataibo ont commencé à produire à l'occasion des réunions politiques, font partie de ce processus de construction d'une nouvelle identité qui se veut à la fois attirante, commerciale et revendicative.

\section{Références citées}

Carneiro Da Cunha, Manuela, 2010. Savoir traditionnel, droits intellectuels et dialectique de la culture. Paris : Éditions de l'éclat.

Cima « Instrumentos de Proteccion para Pueblos Indigenas en aislamiento », 2011.

$<$ http://www.cima.org.pe/adm/upload/descargas/CIMA.2012.\%20cartilla\%20PIAV\%20institucional. pdf $>$

Comaroff, John et Jean Comaroff, 2009. Ethnicity, Inc. Chicago : University of Chicago Press.

Cortez Mondragón, María, 1987. « Yo vi a los Kamano. Habla un Cacataibo llamado Aniceto », Amazonia Peruana, 14, pp. 123-129.

DÉLÉAge, Pierre, 2005. Le chamanisme sharanahua. Enquête sur l'apprentissage et l'épistémologie d'un rituel. Thèse de doctorat soutenue à l'École des Hautes Études en Sciences Sociales, Paris.

Descola, Philippe, 1993. «Les Affinités sélectives. Alliance, guerre et prédation dans l'ensemble jivaro », L'Homme, 33 (126-128), pp. 171-190.

DziUBinsKa, Magda Helena, 2014. «Pas tout le monde sait faire une belle fête. Le prestige incertain du chef Kakataibo (Amazonie péruvienne) », in Frédéric Hurlet, Isabelle Rivoal, Isabelle Sidéra (éds), Le 
prestige. Autour des formes de la différenciation sociale. Actes du colloque de la MAE. Paris : Éditions de Boccard, pp. 57-65.

ERIKSON, Philippe, 1986. «Altérité, tatouage et anthropophagie chez les Pano : la belliqueuse quête du soi », Journal de la Société des Américanistes, 72 (1), pp. 185-210.

-, 1996. La Griffe des aïeux. Marquage du corps et démarquages ethniques chez les Matis d'Amazonie. Paris : Peeters Publishers.

-, 2004. « La face cachée de l'ancestralité. Masques et affinité chez les Matis d'Amazonie brésilienne », Journal de la Société des Américanistes, 90 (1), pp. 119-142.

Fortes, Meyer, 1965. « Some Reflections on Ancestor Worship in Africa », in M. Fortes et G. Dieterlen (éds), Africa System of Thought. Londres : Oxford University Press.

Frank, Erwin, 1990. Mitos de los uni de Santa Marta. Quito : Abya-Yala.

-, 1994. « Los Uni », in Fernando Santos-Granero et Frederica Barclay (éds), Guía Etnográfica de la Alta Amazonia, 2, pp. 133-237. Quito : FLACSO et IFEA.

Galinier, Jacques et Antoinette MoliniÉ, 2006. Les néo-Indiens : Une religion du III ème millénaire. Paris : Éditions Odile Jacob.

Gow, Peter, 1993. "Gringos and Wild Indians Images of History in Western Amazonian Cultures », L'Homme, 33 (126), pp. 327-347.

Instituto Del Bien Comun, 2005. Propuesta de Creación de Dos Reservas Territoriales a Favor de los Cacataibos en Aislamiento ». Aguaytia/Lima.

KeIFENHEIm, Barbara, 1997. «Futurs beaux-frères ou esclaves ? Les Kashinawa découvrent des Indiens non contactés », Journal de la Société des Américanistes, 83, pp. 141-158.

KорутоғF, Igor, 1971. «Ancestors as Elders in Africa », Africa : Journal of the International African Institute, 41 (2), pp. 129-142.

La Torre-Cuadros, María de los Ángeles, 2011. Catálogo Arte Kakataibo. Comunidades Nativas Yamino y Mariscal Cáceres. Lima : World Agroforestry Centre (ICRAF).

SAhLins, Marshall, 2007 [1993]. « Adieux aux Tristes Tropiques. L'ethnographie dans l'histoire du monde moderne », La découverte du vrai Sauvage et autres essais. Paris : Gallimard.

Shell, Olivia, 1973. Cuentos del Hombre Cacataibo (cashibo) y la Obra Civilizadora de Bolivar. Pérou : Summer Institute of Linguistics.

Tessmann, Günter, 1999 [1930]. Los Indigenas Del Perú Nororiental : Investigaciones Fundamentales para un Estudio Sistemático de la Cultura. Quito : Ediciones Abya-Yala.

VILAÇA, Aparecida, 1999. « Devenir autre : chamanisme et contact interethnique en Amazonie brésilienne », Journal de la Société des Américanistes, 85, pp. 239-260.

Viveiros De CAstro, Eduardo, 1992. From the Ennemy's Point of View. Chicago : The University of Chicago Press.

Wistrand, Lila, 1969. Folkloric and Linguistic Analysis of Cashibo Narrative Prose. Thèse de doctorat soutenue à l'Université de Texas, Austin.

ZariQuiey Biondi, Roberto, 2013. « Del 'Kaschibo' de Tessmann al Cashibo-Cacataibo Contemporáneo : Algunas Notas Para la Comprensión de la Historia Lingüística de un Pueblo Pano ». Revista Brasileira de Linguística Antropológica, 5 (1), pp. 159-192. 